\title{
DNA Copy Number Changes in Epithelioid Sarcoma and Its Variants: A Comparative Genomic Hybridization Study
}

Tamara Lushnikova, M.D., Sakari Knuutila, Ph.D., Markku Miettinen, M.D.

Department of Medical Genetics, Haartman Institute and Helsinki University Hospital, University of Helsinki, Helsinki, Finland; Molecular and Cell Biology Institute, University of Tartu, Estonia; Department of Soft Tissue Pathology, Armed Forces Institute of Pathology, Washington DC

Epithelioid sarcoma is a distinctive, rare soft tissue sarcoma that typically involves the distal extremities in young adults, and shows epithelioid morphology and immunohistochemical markers of epithelial differentiation. The genetic background of epithelioid sarcoma is poorly understood, and knowledge of it could give insights into the pathogenesis of this tumor and its possible relationship with other malignant tumors. In this study, we analyzed DNA copy number changes in 30 epithelioid sarcomas by comparative genomic hybridization. DNA was extracted from microdissected samples of formaldehyde-fixed and paraffin-embedded tumors with a minimum of $60 \%$ of tumor cells in each sample. Sixteen tumors $(53 \%)$ showed DNA copy number changes at one to six different genomic sites. The majority of the changes were gains, seen in 14 tumors, whereas 10 tumors showed losses. The most common recurrent gains were at 11 q13 (five cases), 1q21-q23 (four cases), 6p21.3 (three cases), and 9q31-qter (three cases). High-level amplifications were detected once in 6p21.3-p21.1 and once in 9q32-qter. Recurrent losses were seen at 9pterp23 (three cases), 13q22-q32 (three cases), 1p13-p22 (two cases), 3p12-p14 (two cases), 4q13-q33 (two cases), 9p21 (two cases), and 13q32-qter (two cases). The most common recurrent gain at 11 q13 was seen in both classic cases and angiomatoid and rhabdoid variants supporting the relationship of these variants with the classic epithelioid sarcoma. Expression of cyclin D1 gene, located in 11q13, was immunohistochemically detected in nine of 15 cases

Copyright $(\mathcal{C} 2000$ by The United States and Canadian Academy of Pathology, Inc.

VOL. 13, NO. 10, P. 1092, 2000 Printed in the U.S.A.

Date of acceptance: April 11, 2000.

The opinions and assertions contained herein are the expressed views of the authors and are not to be construed as official or reflecting the views of the Departments of the Army or Defense.

Address reprint requests to: Markku Miettinen, M.D., Armed Forces Institute of Pathology, Department of Soft Tissue Pathology, 14th Street \& Alaska Avenue, N.W., Washington, DC 20306-6000; fax: 202-782-9182. including three of five cases with gain of 11q13, suggesting its involvement in epithelioid sarcoma. The observed comparative genomic hybridization changes give targets for future genetic studies on epithelioid sarcoma.

KEY WORDS: Cyclin D1, Epithelioid sarcoma, Genetics.

Mod Pathol 2000;13(10):1092-1096

Epithelioid sarcoma, first described by Enzinger in 1970 , is a rare soft tissue sarcoma typically presenting in young adults in distal parts of the extremities (1). Occurrence in more proximal body sites has also been documented in rare cases (2). The patients often develop multiple local recurrences, and lymph node, soft tissue, and pulmonary metastases eventually develop in 30 to $50 \%$ of the patients (3-4).

Histologically, epithelioid sarcoma shows distinctive clusters of epithelial-like tumor cells with abundant, eosinophilic cytoplasm and immunohistochemical markers of epithelial differentiation: keratins and epithelial membrane antigen, and CD34 in 50\% of the cases (5-10). The histogenesis of epithelioid sarcoma is unknown, but origin from mesenchymal cells undergoing epithelial differentiation has been favored.

Comparative genomic hybridization ( $\mathrm{CGH}$ ) is a powerful method that allows for the study of genomewide DNA copy number changes in the tumor DNA in comparison with differentially labeled normal DNA hybridized on normal metaphases. This method makes it possible to analyze DNA losses, gains, and amplifications. The areas of loss may involve tumor suppressor genes, whereas gains and amplifications may involve oncogenes (11-14).

Comparative genomic hybridization of epithelioid sarcoma is of particular interest because, due to its rarity, the cytogenetic information on epithelioid sarcoma is limited to isolated case studies. 
Losses or structural rearrangements have been detected in $1 \mathrm{p}$ (15), and structural rearrangements have been found in 18q (16). A balanced translocation involving $\mathrm{t}(8 ; 22)(\mathrm{q} 22 ; \mathrm{q} 11)$ has been reported (17).

\section{MATERIALS AND METHODS}

\section{Tissue Material}

Paraffin-embedded tissue from 30 epithelioid sarcomas was obtained from the soft tissue registry of the Armed Forces Institute of Pathology. All epithelioid sarcomas were histologically and immunohistochemically characterized. The sampling for comparative genomic hybridization was performed by microdissecting clusters of tumor cells from the paraffin-embedded tissue, using sharp razor blades. The dissected pieces were histologically evaluated, and were found to contain at least 60 to $70 \%$ of tumor cells in each specimen.

\section{DNA Samples}

Tumor DNA isolation from formalin-fixed paraffin-embedded tissue sections was prepared as previously described (18).

\section{Comparative Genomic Hybridization}

CGH was performed using direct fluorochromeconjugated DNAs according to a published protocol (19). Tumor and normal reference DNAs were labeled by nick translation with fluorescein 12-dUTP and Texas Red-5-dUTP (DuPont, Boston, MA). Fluorochrome-dCTP and fluorochrome-dUTP were mixed 1:1. DNA fragments of 500 to $2000 \mathrm{bp}$ were obtained. The hybridization mixture consisted of $1000 \mathrm{ng}$ of tumor DNA, $1000 \mathrm{ng}$ of normal labeled DNA, and $20 \mu \mathrm{g}$ of unlabeled human Cotl DNA (Gibco BRL, Life Technologies, Gaithersburg, MD), dissolved in $10 \mu \mathrm{L}$ of hybridization buffer (50\% formamide, $10 \%$ dextran sulfate, $2 \times \mathrm{SSC}$ ). The mixture was denatured at $75^{\circ} \mathrm{C}$ for $5 \mathrm{~min}$ and then hybridized onto normal metaphases denatured in $70 \%$ formamide $/ 2 \times \mathrm{SSC}$ at $65^{\circ} \mathrm{C}$ for $2 \mathrm{~min}$. Hybridization were carried out at $37^{\circ} \mathrm{C}$ for 48 hours. The slides were washed three times in $50 \%$ formamide/ $2 \times$ SSC (pH7.0), twice in $2 \times$ SSC, and once in $1 \times$ SSC (at $45^{\circ} \mathrm{C}$ for $10 \mathrm{~min}$ each), followed by washes in $2 \times$ SSC in a buffer containing $0.1 \mathrm{~mol} / \mathrm{L}$ $\mathrm{NaH}_{2} \mathrm{PO}_{4}$ and $0.1 \mathrm{mo} / \mathrm{L} \mathrm{Na}_{2} \mathrm{HPO}_{4}$ (pH8.0) and in distilled water (at room temperature for $10 \mathrm{~min}$ each). The slides were counterstained with DAPI and mounted with Vestashield antifading buffer (Vector Laboratories).

\section{Digital Image Analysis}

The hybridization was analyzed using an Olympus fluorescence microscope and the ISIS digital image analysis system (MetaSystem Hard\&Software, Altlussheim, Germany). Three-color images, green (fluorescein isothiocyanate) for the tumor DNA, red (Texas Red) for the reference DNA, and blue (DAPI) for the chromosome counterstain, were obtained. The areas were considered to be over-represented when the green-to-red ratio was higher 1.17 (gains) or 1.5 (highlevel amplifications, more than 10-fold), and underrepresented when the ratio was lower than 0.85 (losses). Telomeric and heterochromatic regions were excluded from the analysis when they appeared as the sole aberration present in the sample as these regions cannot be evaluated reliably by CGH.

\section{Immunohistochemistry}

Immunohistochemical analysis for keratin 8 (CAM 5.2, Beckton-Dickinson, Mt. View, CA, 1:40), keratin 19 (RCK108, Dakopatts, Carpinteria, CA, 1:50), epithelial membrane antigen (E29, Dakopatts, 1:40), CD34 (HPCA1, Beckton-Dickinson, 1:40), and cyclin D1 (5D4, Immunotech, Westbrook, ME, 1:3000) were performed by immunoperoxidase technique using the $\mathrm{ABC}$-detection system with diaminobenzidine as the chromogen. Enzyme digestion $\left(0.05 \%\right.$ pepsin for $30 \mathrm{~min}$ at $\left.37^{\circ} \mathrm{C}\right)$ was used for keratin 8 , and heat-induced epitope retrieval was used for CD34, EMA, keratin 19, and cyclin D1. Positive and negative controls were included.

\section{RESULTS}

\section{Clinical Features}

The patients' ages ranged from 15 to 62 years (median, 26 years; mean, 33 years). There were 22 males and 8 females (male to female ratio, 2.75). The most common locations of the tumors in descending order were: hand and wrist, eight; foot, four; forearm, three; shoulder, three; arm, two; inguinal, two; leg, two; and one each in the buttock, chest wall, perineum, scalp, thigh, and vulva. The tumor size was often difficult to determine because of the complex tumor involvement along the tendon sheath and fascia. However, the measurable tumors in hand measured from 1.5 to $3.0 \mathrm{~cm}$, whereas the three largest tumors in the thigh, inguinal area, and chest wall measured $8 \mathrm{~cm}, 9 \mathrm{~cm}$, and $17 \mathrm{~cm}$, respectively, in maximum diameter.

\section{Histologic and Immunohistochemical Features}

Twenty-five cases were typical variants showing clusters of epithelioid tumor cells with strongly eosinophilic cytoplasm surrounded by spindle cells stroma. Three cases represented the angiomatoid variant showing clusters of tumor cells lining pseudoangiomatoid spaces filled with erythrocytes. Two cases showed rhabdoid features with large cy- 
toplasmic tumor cells showing rounded, eosinophilic inclusion-like cytoplasm. Keratin 8 (CAM 5.2) was demonstrated in 29 of 30 cases, and keratin 19 in 17 of 28 cases. Most cases (22/24) were positive for epithelial membrane antigen. CD34 was detected in 12 of 26 cases that were analyzed.

\section{Overview of DNA Copy Number Changes}

Sixteen of the 30 cases (53\%) showed DNA copy number changes. These cases showed one to six aberrations each, whereas 14 cases with successful hybridization showed no DNA copy number changes.

In the 16 cases with copy number changes, gains were more frequent than losses (gains:losses, 1.6:1). All chromosomal regions with an increase (gains) or decrease (losses) in DNA copy number changes are shown in Figure 1, and the most common changes have been summarized in Table 1 .

\section{Gains and High-Level Amplifications}

Fourteen cases showed gains. The most common recurrent gains were at 11q13 (five cases), 1q21-q23 (four cases), 6p21.3 (three cases), and 9q31-qter (three cases). High-level amplifications were observed on two areas that showed recurrent gains. One case showed amplification of 6p21.3-p21.1, and another case showed an amplification at 9q32-qter.

\section{Losses}

Ten cases showed losses. Recurrent losses were detected at 9pter-p23 and 13q22-q32 (both in three cases). Two cases showed losses in 1p13-p22, 3p1214, 4q13-q33, 9p21, and 13q32-qter.

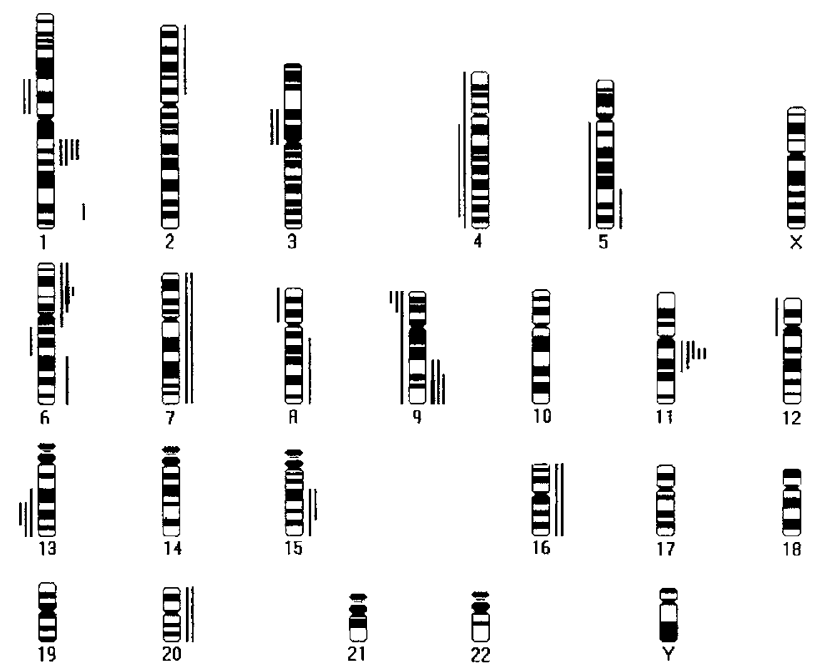

FIGURE 1. Summary of the DNA copy number changes detected in 16 epithelioid sarcomas. The bars on the left side of the chromosome indicate losses, and the bars on the right side indicate gains. High level amplifications are marked by thick bars (at 6p21.3 and 9q32-qter).
Cyclin D1 Expression and Its Relationship with Amplification in 11q13

Cyclin D1 was immunohistochemically detected in the tumor cell nuclei in nine of 15 cases; 25 to $60 \%$ of tumor cells showed nuclear positivity (Fig. 2). Three of the five cases showed a coincidence of expression of cyclin D1 and gain of 11q13.

\section{Occurrence of Changes in Special Subtypes of Epithelioid Sarcoma}

DNA copy number changes occurred in two of the three angiomatoid and in both rhabdoid/large cell variants of epithelioid sarcoma that were studied. The gain in 11q12-21 occurred in one of the angiomatoid in one of the two large cell/rhabdoid variants. One of the high level amplifications, at 6p21.3-p21.1, occurred in an angiomatoid variant.

\section{Possible Relationship with DNA Copy Number Changes and Survival}

Of the thirteen patients who were confirmed to be alive, five patients (38\%) had DNA copy number changes, whereas four of the five patients $(80 \%)$ who were dead had DNA copy number changes. Because of the small number of observations, the individual changes were not evaluated in this respect.

\section{DISCUSSION}

CGH is probably the most powerful method currently available to survey DNA copy number changes in fixed tissue. This method is highly useful to find involved areas that are of interest for further studies. Recurrent changes may sometimes be characteristic as tumor type markers, or they may point into areas that are of interest for $\mathrm{LOH}$ studies or evaluating changes in specific candidate genes.

In this study, we analyzed DNA sequence copy number changes in 30 cases of epithelioid sarcomas. To our knowledge, this is the first study to apply CGH on epithelioid sarcomas. This method allows for the detection of the DNA gains and losses in particular areas of the genome, and gives an overview of such changes with the resolution of chromosomal microscopy (11-13). Several recurrent gains and losses were detected.

The epithelioid sarcomas analyzed in this study were clinicopathologically and morphologically representative examples of this entity. Similar to the largest published series (3), the tumors in this study predominantly occurred in distal extremities and had a significant male predominance. Also the immunohistochemical features were typical, including the consistent expression of keratins and EMA and the presence of CD34 in half of the cases (8-10). 
TABLE 1. Minimal Overlapping Areas in Most Frequent Gains, High-Level Amplifications, and Losses of DNA Sequence Copy Numbers as Detected by CGH in Epithelioid Sarcomas

\begin{tabular}{|c|c|c|c|c|c|}
\hline \multicolumn{2}{|c|}{ Gains } & \multicolumn{2}{|c|}{ High-Level Amplification } & \multicolumn{2}{|c|}{ Losses } \\
\hline Location & Number of Cases & Location & Number of Cases & Location & Number of Cases \\
\hline $11 q 13-q 14$ & 5 & 6p21.3-p21.1 & 1 & 9pter-p23 & 3 \\
\hline 1q21-q23 & 4 & $9 q 32$ & 1 & $13 q 22-q 32$ & 3 \\
\hline $6 \mathrm{p} 21.3$ & 3 & & & 1p13-p22 & 2 \\
\hline \multirow[t]{4}{*}{ 9q31-qter } & 3 & & & 3p12-p14 & 2 \\
\hline & & & & $4 q 13-q 33$ & 2 \\
\hline & & & & $9 \mathrm{p} 21$ & 2 \\
\hline & & & & 13q32-qter & 2 \\
\hline
\end{tabular}

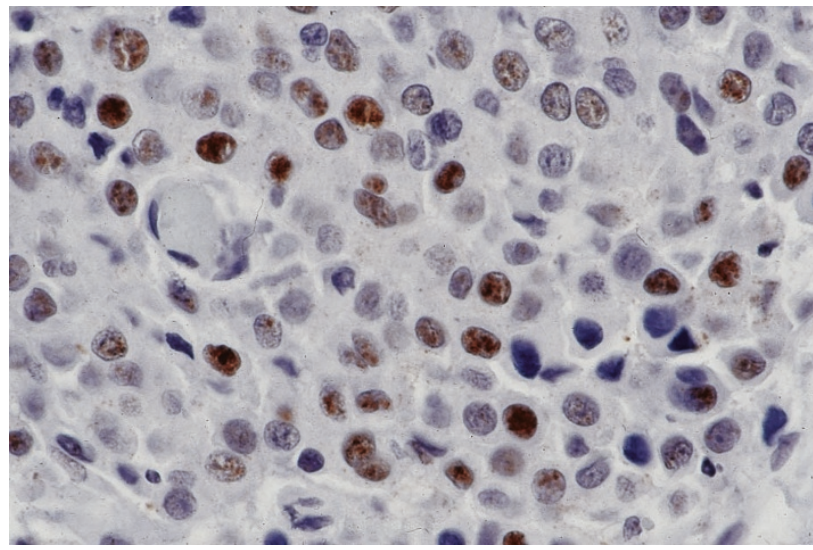

FIGURE 2. Approximately $50 \%$ of the nuclei of this epithelioid sarcoma show cyclin D1 immunoreactivity.

Sixteen of the 30 epithelioid sarcomas analyzed (53\%) showed DNA sequence copy number changes. The lack of changes in half of the cases indicates a common lack of detectable copy number changes in epithelioid sarcoma. The results therefore suggest that the genetic changes in many epithelioid sarcomas may be limited to balanced translocations, specific mutations, or other submicroscopic gene rearrangements that are undetectable by comparative genomic hybridization. Similarly, two studies on DNA content by flow cytometry determined that the majority of epithelioid sarcomas were diploid or near diploid (20-21), although a third study found aneuploidy in $64 \%$ of the tumors (22). Although DNA flow cytometry only reveals net changes in the overall DNA content, diploid findings in these studies may also suggest that the many epithelioid sarcomas have DNA alterations more delicate than copy number changes.

The possibility cannot be ruled out that in some of the cases, infiltrating normal cells (lymphocytes, fibroblastic background) may have diluted the DNA pool and made the DNA copy number changes undetectable in CGH (or flow cytometry) analysis. Although we used histologically correlated microdissection in the sampling to yield as pure tumor cell population as possible, the cellular heterogeneity of epithelioid sarcoma tissue may pose a sampling problem for genetic studies.
The 16 of 30 epithelioid sarcomas (53\%) with CGH changes had one to six DNA copy number changes each. Gains, some of them of whole chromosomes or chromosome arms, were more frequent than losses. High-level amplifications were detected in two cases in two different regions.

The most common recurrent change was gain in band 11q13 that was found in five cases $(31 \%$ of the cases with DNA copy number changes). Gains in this region have been found with in several types of carcinomas, including those of respiratory tract (23) and breast (24). Such gains have also been found in glioblastoma multiforme (25). Cyclin D1 gene is located in the 11q13 region and has been found overexpressed and important in oncogenesis of several tumor types including mantle cell lymphoma, where it is activated by translocation (26), and in breast carcinoma where it is upregulated by an unknown mechanism (27).

Our immunohistochemical studies on cyclin D1 revealed nuclear expression in the majority of cases that were studied, and the observed coincidence of cyclin D1 expression and gain of 11q13 in three cases may suggest that cyclin D1 gene may be amplified and overexpressed in epithelioid sarcoma. However, the correlation was not complete, as two cases with amplification lacked detectable expression. Additional studies are needed to evaluate the role of the cyclin D1 gene alterations in epithelioid sarcoma.

Other oncogenes known to be amplified and overexpressed in carcinomas with amplification of 11q13 include EMS1 encoding cytoskeleton-associated signal transduction protein cortactin (28) and fibroblast growth factors FGF-3 (INT) and FGF-4 (29). Whether these oncogenes have a specific role in epithelioid sarcoma needs to be investigated.

The gains in 1q21-q23 were seen in four cases ( $27 \%$ of the cases with DNA copy number changes). Genes located in this region, in 1q21, and known to be amplified in human sarcomas include FLG (filaggrin), NTRK1 (neurotrophic tyrosine kinase receptor, type 1), and SPRR3 (small proline rich protein 3 ), according to the genome database.

Among the areas of DNA copy number losses, $9 p$ is known to include MTS1, MTS2, and cyclin dependent kinase 2 (CDNK2). MTS1 and MTS2 both en- 
code cyclin-dependent kinase 4 inhibitors (p16 and p15) at 9p21. Losses of DNA sequence on $13 q$ and 3p12-14 suggest that these regions might harbor tumor suppressor genes relevant to the tumorigenesis of epithelioid sarcoma.

Five variant epithelioid sarcomas were studied. Two of the three angiomatoid epithelioid sarcomas and both large cell/rhabdoid variants contained DNA copy number changes, including gain of $11 q 13$ in one case of each variant. The apparently higher frequency of DNA copy number changes in the variant tumors (four of five cases) suggests the possibility that such variants may reflect a more genetically advanced disease. However, more cases should be studied to determine if there are genetic differences among the variants of epithelioid sarcoma. It also appears, that the presence of any copy number changes might have adverse prognostic significance because the overall occurrence of changes was less common in survivors (38\%) than in those who died of disease $(80 \%)$.

In summary, our comparative genomic hybridization study on 30 epithelioid sarcomas showed changes in slightly over half of the cases, including recurrent gains in 11q23 in five cases (some of them with cyclin D1 overexpression). The present results outline areas of losses that should be evaluated by LOH (loss of heterozygosity) studies or gains that should be further explored for the amplification or other involvement of specific candidate genes.

\section{REFERENCES}

1. Enzinger FM. Epithelioid sarcoma. A sarcoma simulating granuloma or a carcinoma. Cancer 1970;26:1029-41.

2. Guillou L, Wadden C, Coindre J-M, Krausz T, Fletcher CDM. "Proximal-type" epithelioid sarcoma, a distinctive aggressive neoplasm showing rhabdoid features. Clinicopathological, immunohistochemical and ultrastructural study of a series. Am J Surg Pathol 1997;21:130-46.

3. Chase DR, Enzinger FM. Epithelioid sarcoma. Diagnosis, prognostic indicators, and treatment. Am J Surg Pathol 1985;9:241-63.

4. Halling AC, Wollen PC, Pritchard DJ, Vlasak R, Nascimento AG. Epithelioid sarcoma. A clinicopathologic review of 55 cases. Mayo Clin Proc 1996;71:636-42.

5. Chase DR, Weiss SW, Enzinger FM, Langloss J. Keratin in epithelioid sarcoma. An immunohistochemical study. Am J Surg Pathol 1984;8:435-41.

6. Mukai M, Torikata C, Iri H, Hanaoka H, Kawai T, Yakumaru $\mathrm{K}$, et al. Cellular differentiation of epithelioid sarcoma. An electron-microscopic, enzyme histochemical, and immunohistochemical study. Am J Pathol 1985;119:44-56.

7. Daimaru Y, Hashimoto H, Tsuneoshi M, Enjoji M. Epithelial profile of epithelioid sarcoma. An immunohistochemical analysis of eight cases. Cancer 1987;59:131-41.

8. Manivel JC, Wick MR, Dehner LP, Sibley RK. Epithelioid sarcoma. An immunohistochemical study. Am J Clin Pathol 1987;87:319-26.

9. Arber DA, Kandalaft PL, Mehta P, Battifora H. Vimentin-negative epithelioid sarcoma. The value of an immunohistochemical panel that includes CD34. Am J Surg Pathol 1993;17:302-7.

10. Miettinen M, Fanburg-Smith J, Virolainen M, Shmookler BM,
Fetsch JF. Epithelioid sarcoma: an immunohistochemical analysis of 112 classical and variant cases and a discussion of the differential diagnosis. Hum Pathol 1999;30:934-42.

11. Kallioniemi A, Kallioniemi O-P, Sudar D, Rutovitz D, Gray JW, Waldman F, et al. Comparative genomic hybridization for molecular cytogenetic analysis of solid tumors. Science 1992;4:818-21.

12. Knuutila S, Bjorkqvist A-M, Autio K, Tarkkanen M, Wolf M, Monni $\mathrm{O}$, et al. DNA copy number amplifications in human neoplasms: review of comparative genomic hybridization studies. Am J Pathol 1998;152:1107-23.

13. Knuutila S, Aalto Y, Autio K, Björkqvist A-M, El-Rifai W, Hemmer S, et al. DNA copy number losses in human neoplasms. Am J Pathol 1999;155:683-94.

14. Hermsen MA, Meijer GA, Baak JP, Joenje H, Walboomers JJ. Comparative genomic hybridization: a new tool in cancer pathology. Hum Pathol 1996;27:342-9.

15. Stenman S, Kindblom LG, Willems J, Angervall L. A cell culture, chromosomal and quantitative DNA analysis of a metastatic epithelioid sarcoma. Cancer 1990;65:2006-13.

16. Iwasaki H, Ohjimi Y, Ishiguro M, Isayama T, Kaneko Y, Yoh S, et al. Epithelioid sarcoma with an 18q aberration. Cancer Genet Cytogenet 1996;91:46-52.

17. Cordoba JC, Parham DM, Meyer WH, Douglas EC. A new cytogenetic finding in an epithelioid sarcoma t(8;22)(q22; q11). Cancer Genet Cytogenet 1994;72:151-4.

18. Isola J, DeVries S, Chu L, Ghazvini S, Waldman F. Analysis of changes in DNA sequence copy number by comparative genomic hybridization in archival paraffin-embedded tumor samples. Am J Pathol 1994;145:1301-7.

19. El-Rifai W, Larramendy ML, Bjorkqvist A-M, Hemmer S, Knuutila S. Optimization of comparative genomic hybridization using fluorochrome conjugated to dCTP and dUTP nucleotides. Lab Invest 1997;77:699-700.

20. el-Naggar AK, Garcia GM. Epithelioid sarcoma. Flow cytometric study of DNA content and regional DNA heterogeneity. Cancer 1992;69:1721-8.

21. Ishida T, Oka T, Matsushita H, Machinami R. Epithelioid sarcoma: an electron microscopic, immunohistochemical and DNA flow cytometric analysis. Virchows Arch A Pathol Anat Histopathol 1992;421:401-8.

22. Pastel-Levy C, Bell DA, Rosenberg AE, Preffer F, Colvin RB, Flotte TJ. DNA flow cytometry in epithelioid sarcoma. Cancer 1992;70:2823-6.

23. Petersen I, Langreck H, Wolf G, Schwendel A, Psille R, Vogt P, et al. Small-cell lung cancer is characterized by a high incidence of deletions on chromosome 3p, 4q, 5q, 10q, 13q and 17p. Br J Cancer 1997;75:79-86.

24. Courjal F, Theillet C. Comparative genomic hybridization analysis of breast tumors with predetermined profiles of DNA amplification. Cancer Res 1997;57:4368-77.

25. Weber RG, Sommer C, Albert FK, Kiessling M, Cremer T. Clinically distinct subgroups of glioblastoma multiforme studied by comparative genomic hybridization. Lab Invest 1996;74:108-19.

26. Campo E, Raffeld M, Jaffe ES. Mantle cell lymphoma. Semin Hematol 1999;36:115-27.

27. Jares P, Rey MJ, Fernandez PL, Campo E, Nadal A, Munoz M, et al. Cyclin D1 and retinoblastoma gene expression human breast carcinoma: correlation with tumour proliferation and oestrogen receptor status. J Pathol 1997;182:160-6.

28. Schuuring E, van Damme H, Schuuring-Scholtes E, Verhoeven E, Michaelides R, Geelen E, et al. Characterization of the EMS1 gene and its product, human cortactin. Cell Adhes Commun 1998;6:185-209.

29. Schmitt JF, Susil BJ, Hern MT. Aberrant FGF-2, FGF-3 FGF-4 and $C$-erb-B2 gene copy number in human ovarian, breast and endometrial tumors. Growth Factors 1996;13:19-35. 